\section{Not all cultural traditions deserve respect}

\author{
Ruth Macklin
}

The position Arora and Jacobs defend regarding female genital alteration (FGA) has much to recommend it. Sanctioning a form of FGA that seeks to minimise if not eliminate harm to infants, adolescents and adult women, and at the same time show respect for cultural traditions appears to make good sense. In arguing for a de minimis procedure, the authors contend that any harm would be equivalent to that of male circumcision, a practice that is permitted by countries that have made FGA illegal. They are correct in saying that in a de minimis form, FGA could not reasonably be considered a human rights violation.

With these and other reasons that seem persuasive at first blush, why do I remain resistant to accepting this apparent solution to a public health problem that affects millions of girls and women? Two different considerations lead me to reject the proposal. The first might be dismissed as 'merely symbolic', but is nevertheless real. The second is deep scepticism regarding several empirical premises that underlie the authors' position.

There is no doubt that in whatever form, FGA has its origin and purpose in controlling women. Whether it be controlling their sexual behaviour in the most extreme form by sewing up the vaginal opening, or the lesser version of clitorodectomy to eliminate women's sexual pleasure, or the social requirement of making it a condition of being marriageable, as a cultural rite it signifies a means of making girls and women physically, aesthetically or socially acceptable to men. Arora and Jacobs contend that a minimally invasive form of FGA has parity with male circumcision tolerated in liberal societies. That may be true regarding the degree of harm the procedure causes, but it is not true of the origins or the continued symbolic meaning of FGA as a necessity for being an 'acceptable' woman. Those who would dismiss this concern as 'merely symbolic' should reflect on the recent controversy in the southern state of South Carolina in the USA. After a 21-year-old racist committed a hate crime, shooting and killing nine African-Americans in a church, debate ensued about removing the Confederate flag from the statehouse. The

Correspondence to Professor Ruth Macklin, Albert Einstein College of Medicine, 1300 Morris Park Avenue, Bronx, NY 10461, USA; ruth.macklin@einstein.yu.edu debate continues in numerous southern US states about whether to remove the flag and other symbols of the confederacy, which fought the Civil War to retain the system of slavery. Although it may be a 'mere symbol', displaying the flag today signifies overt racism, whatever its defenders may claim about cultural tradition in the southern USA. Not all cultural symbols deserve respect.

As for the questionable empirical premises, Arora and Jacobs admit that they are 'not suggesting that people whose beliefs or sense of propriety leads them to perform these procedures on their children would necessarily accept alterations in their practices to conform to the authors' views of what is acceptable'. (p. 4) Based on some of the statistics they cite, evidence points in the opposite direction. In Somalia, for example, a study revealed that $81 \%$ of subjects underwent infibulation and only $3 \%$ did not have FGA. 'Eighty-five per cent had an intention to subject their daughters to an extensive FGA procedure, and 90\% supported the continuation of the practice'. (p. 2) The example cited of the Seattle hospital in which a compromise was reached with the local Somali population to allow a 'ritual nick' is hardly convincing evidence of what is likely to take place in Somalia. Whether it is $90 \%$ or a lesser percentage of the population, defenders of the most invasive form of FGA could readily maintain that respect for their culture requires toleration of the procedure they favour. The question of precisely what 'cultural sensitivity' allows or requires has no satisfactory answer. Like other vague concepts, it is used to mean exactly what the speaker wants it to mean in a given context.

The authors point out that 'marriage is associated strongly with quality of life in these traditions. In some cultural milieus, a woman who has not undergone a procedure to alter her external genitalia may find it difficult to marry'. (p. 14) This prompts the question how verification takes place in current practice, and how it can take place when the authors' proposed procedure is used. In arguing against using the term 'mutilation' to refer to FGA, the authors say 'a nick that heals completely is not mutilation in that there is no morphological alteration'. (p. 7)
When the ritual is performed in infancy or even puberty and 'heals completely', it may be difficult to detect at the time of marriage. When FGA is conducted as part of a ceremony, whether at birth, as a rite of passage in puberty or in preparation for marriage, it is a community event. In addition to the individual who does the procedure, relatives of the girl or woman are present and there are witnesses, who may include members of the groom's family. But if the procedure is done by a medical professional in a hygienic setting, who will be present? And what form of verification is envisaged if FGA remains a condition for marriage? As one article notes: 'the intrusion of the groom and his family takes place even before he has married the bride, it occurs prior to the marriage proposal. The prospective groom may claim his right to ascertain that the woman is a virgin by inspecting her infibulation scar' (ref. ${ }^{1}$, note 59, p.416). It is demeaning, to say the least, to require women to undergo inspection of their genitalia if FGA in whatever form is a cultural requirement for marriage.

The authors cite evidence that the prevalence of FGA is decreasing in some countries, especially among younger women. Cultural change proceeds slowly. But with strong support from nongovernmental organisations, especially those comprising local and regional women, a cultural tradition designed to control women-even in its least harmful form-is best abandoned.

\section{Competing interests None declared.}

Provenance and peer review Not commissioned; internally peer reviewed.

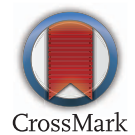

To cite Macklin R. J Med Ethics 2016;42:155.

Received 30 July 2015

Accepted 23 August 2015

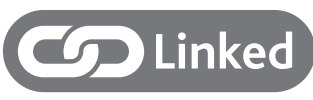

- http://dx.doi.org/10.1136/medethics-2014-102375

- http://dx.doi.org/10.1136/medethics-2015-103029

- http://dx.doi.org/10.1136/medethics-2015-103030

- http://dx.doi.org/10.1136/medethics-2016-103376

J Med Ethics 2016;42:155

doi:10.1136/medethics-2015-103027

\section{REFERENCE}

1 Sharif KF. Female genital mutilation: what does the new federal law really mean? Fordham Urban Law J 1996;24(2):408-26. 